\title{
Risk factors associated with malaria infection among pregnant women of African Descent in Specialist Hospital Sokoto, Nigeria
}

\begin{abstract}
Background: Malaria in pregnancy is a major contributor to adverse maternal and prenatal outcome. In hyper endemic areas like ours, it is a common cause of anaemia in pregnancy and is aggravated by poor socioeconomic circumstance. This study evaluated the sociodemographic risk factors associated with malaria infection among Pregnant Women of African Descent in Specialist Hospital Sokoto, Nigeria.
\end{abstract}

Main body: A total of 60 consecutively recruited malaria- positive pregnant women participated in the study. Participants were recruited from the antenatal Clinic of Specialist Hospital Sokoto. A structured interviewer-administered questionnaire was used to obtain some socio-demographic characteristics. Data generated was analyzed using SPSS 25.0 statistical package. A p-value $\leq 0.05$ was considered significant in all statistical comparisons. The predominant plasmodium specie responsible for all cases of malaria among the subjects was Plasmodium falciparum. Malaria prevalence was compared based on some sociodemographic factors among the subjects. Subjects residing in urban areas were more prone to malaria compared to those living in rural areas $(X 2=4.957, \mathrm{p}=0.026)$. Pregnant women with no formal education were more predisposed to malaria infection compared to more educated women with primary, secondary and tertiary education $(X 2=9.040, \mathrm{p}=0.029)$. Pregnant women who did not use insecticide- treated mosquito net was more predisposed to malaria compared to those that do $(X 2=32.105, \mathrm{p}=0.0000)$. Pregnant women who are not on prophylactic antimalarial medication were more likely to have malaria compared to those that do $(X 2=18.281, \mathrm{p}=0.0000)$.

Conclusion: This study has shown that Plasmodium falciparum is the predominant malaria specie among plasmodium parasitized women in Sokoto, North Western Nigeria. Sociodemographic factors (residence, illiteracy, non-availability and non -compliance to use of long-lasting insecticide-treated mosquito nets and lack of access to antimalaria prophylaxis during pregnancy) are factors contributing to prevalence of malaria among pregnant African women. There is need to routinely test pregnant women presenting to antenatal clinic particularly with febrile illness for malaria. There is need for the Nigerian government to invest in girl child education and increased awareness about malaria preventive measures, early and regular antenatal clinic attendance as well as universal provision of long insecticide treated mosquito nets and prophylaxis for all pregnant women to reduce the incidence of malaria and its associated morbidities and mortalities.

Keywords: socio-demography, risk factors, malaria, pregnancy, women, African Descent, Specialist Hospital, Sokoto, Nigeria
Volume 10 Issue 4 - 2019

\author{
Erhabor Osaro,' Abdulrahaman Abdullahi,' \\ Erhabor Tosan, ${ }^{2}$ Adias Teddy Charles ${ }^{3}$ \\ 'Department of Haematology, School of Medical Laboratory \\ Science, Usmanu Danfodiyo University Sokoto, Nigeria \\ ${ }^{2}$ Medical Laboratory Science Council of Nigeria, Nigeria \\ ${ }^{3}$ Federal University Otuoke, Nigeria
}

\begin{abstract}
Correspondence: Prof. Osaro Erhabor, Department of Haematology, Usmanu Danfodiyo University Sokoto, Nigeria, School of Medical Laboratory Science, Usmanu Danfodiyo University Sokoto, Nigeria, Tel +447932363217, Email n_osaro@yahoo.com
\end{abstract}

Received: May 27, 2019 | Published: July 18, 2019
Abbreviations: LLINs, long-lasting insecticide-treated nets; IRS, indoor residual spraying; IPTp, intermittent preventive therapy for pregnant women; ACT, artemisinin-based combination therapy; LLITNs, long- lasting insecticide-treated nets; WHO, World Health Organization; ITNs, insecticide-treated nets; IPTp-SP, intermittent preventive treatment with sulfadoxine-pyrimethamine during pregnancy; SP, sulfadoxine-pyrimethamine

\section{Introduction}

An estimated 30 million women living in malaria endemic areas of Africa become pregnant each year. ${ }^{1}$ Pregnant women are particularly vulnerable to malaria because pregnancy reduces immunity to malaria and increases susceptibility to malaria infection. Pregnant women are three times more predisposed to malarial infection compared with their non-pregnant counterparts, and have a mortality rate that approaches $50 \%{ }^{2}$

Malaria in pregnancy is associated with adverse pregnancy outcomes in the mother, her foetus and the newborn. ${ }^{3}$ It has several negative including; maternal anaemia, low birth weight, Malaria in pregnancy is associated with severe anaemia, acute pulmonary oedema, renal failure, puerperal sepsis, postpartum haemorrhage and increased risk of death. Malaria in pregnancy is associated with adverse pregnancy outcomes, such as spontaneous abortion, neonatal death, low birth weight, poor development, behavioural problems, short stature, neurological deficits. ${ }^{4}$

In 2013, there were approximately 198 million cases and 584,000 deaths. ${ }^{5}$ It was estimated that one death occurs each 30 seconds with 
$90 \%$ of malaria deaths. ${ }^{6}$ Nigeria is among the 45 countries that are endemic for malaria with a significant $97 \%$ of the population being at risk especially children and pregnant women. ${ }^{5}$

The increased risk of malarial infection among pregnant women has been linked to a number of sociodemographic factors; illiteracy, young maternal age, low educational status, unemployment and low income. $^{7-9}$

The aim of this study is to investigate the risk factors associated with malaria infection among Pregnant Women of African Descent in Specialist Hospital Sokoto, Nigeria.

\section{Materials and methods}

\section{Study area}

This study was carried out in the Antenatal Clinic of Specialist Hospital, Sokoto, North-Western Nigeria. Specialist Hospital Sokoto is a tertiary institution located within the Sokoto metropolis. Sokoto is the capital city of Sokoto State of Nigeria. The State is located in the extreme Northwest of Nigeria, near to the confluence of the Sokoto River and the Rima River. The State is in the dry Sahel, surrounded by sandy savannah and isolated hills, with an annual average temperature of $28.3^{\circ} \mathrm{C}\left(82.9^{\circ} \mathrm{F}\right)$. Sokoto is, on the whole, a very hot area. However, maximum daytime temperatures are for most of the year generally under $40^{\circ} \mathrm{C}\left(104.0^{\circ} \mathrm{F}\right)$ and the dryness makes the heat bearable. The warmest months are February to April when day time temperature can exceed $45^{\circ} \mathrm{C}\left(113.0^{\circ} \mathrm{F}\right)$. The rainy season is from June to October during which shower are a daily occurrence. Sokoto city is a major commerce center in leather crafts and agricultural products. As at 2006, the state has a population of 3.6 million. ${ }^{10}$ However, based on the population annual growth of $3 \%$, the calculated projected population for Sokoto State now stands at around 4.9 million.

\section{Study population}

The study population for this study includes 60 malaria- infected pregnant women (subject) and 30 age- matched healthy non-parasitized pregnant women who were monitored as controls. Both subjects and controls ware recruited in the Antenatal Clinic of Specialist Hospital, Sokoto, Sokoto North-Western Nigeria.

\section{Study subjects/selection}

\section{Inclusion criteria}

Women who meet the following inclusion criteria were recruited in the study;

a) Adult Pregnant women ( $\geq 18$ years) parasitized with plasmodium attending Antenatal Clinic Specialist Hospital, Sokoto, Nigeria.

b) Women in (a) who gave written informed consent in their clinic and agreed to be included in the study.

\section{Exclusion criteria}

The following were excluded from participating as subjects in the study.

a) Non-adult pregnant women parasitized with plasmodium.

b) Healthy pregnant women that are not parasitized with plasmodium. c) Plasmodium parasitized pregnant women who did not offer an informed consent to be included in the study.

\section{Study design}

The research was a case-control study to assess the effect of malaria on some Haematological and Haemostatic Parameters of 60 Plasmodium parasitized pregnant women and 30 age and gendermatched healthy non-parasitized pregnant women were monitored as controls visiting the Antenatal Clinic Specialist Hospital, Sokoto. Blood sample were collected (from both subjects and controls) and tested for complete blood count, Prothrombin Time and Activated Partial Thromboplastin time.

\section{Sample size determination}

The sample size was determined using the standard formula for calculation of minimum sample size:

$\mathrm{n}=$ minimum sample size

$\mathrm{z}=$ standard normal deviation and probability.

$\mathrm{p}=$ prevalence or proportion of value to be estimated from previous studies.

$\mathrm{q}=$ Proportion of failure (=1-P)

$\mathrm{d}=$ precision, tolerance limit, the minimum is 0.05 .

Therefore, $\mathrm{n}=\mathrm{Z}^{2} \mathrm{pq} / \mathrm{d}^{2}$

Where $Z=95 \%$ (1.96)

$$
\mathrm{P}=6.2 \%(0.062){ }^{.1}
$$

$\mathrm{q}=1-0.062(=0.938)$

$\mathrm{d}=5 \%(0.05)$

Therefore $n=(1.96)^{2}(0.062)(0.938) /(0.05)^{2}$

$\mathrm{n}=88$

\section{Study instrument}

\section{Questionnaire}

A semi- structured interviewer-administered questionnaire was administered to all consenting participants in order to obtain information on their socio-demographic, nutritional and medical history. A sample of the questionnaire is shown in the appendix I.

\section{Informed consent}

Written informed consent was obtained from all the study participants (subjects and controls).

\section{Statistical analysis}

Data obtained was entered into a statistical package (such as SPSS version 25) on a computer to define the nature of the distribution of data for each group. Statistical differences of data were analyzed using series of statistical analysis such as mean, standard deviation, Chi square, student's t-test, ANOVA depending on the nature (categorical or continuous) and distribution of data (normal or non-normal). Pearson's correlation was used to determine the relationship between sets of data. Probability $(p \leq 0.05)$ was used to determine the level of significant for all statistical analysis. 


\section{Ethical consideration}

Ethical approval for this study was obtained from the Ethical Committee of Specialist Hospital Sokoto (Reference number: SHS/ SUB133/VOL.1 $25^{\text {th }}$ May, 2018).

\section{Results}

Table 1 presents the socio-demographic characteristics of the Malaria parasitized pregnant subjects and controls. The prevalence of malaria was significantly higher in pregnant women resident in Urban area, those that do not use mosquito net, those that have no non-formal education and those that are not on prophylaxis medication $(\mathrm{p}<0.05)$. Age, ethnicity and occupation had no significant effect of the prevalence of malaria among pregnant women $(\mathrm{p}>0.05)$. Figure 1 shows the distribution of Malaria parasitaemia based of age. Figure 2 shows the distribution of Malaria parasitaemia among the subjects based on ethnicity. The predominant plasmodium specie responsible for all cases of malaria among the subjects was Plasmodium falciparum. Malaria prevalence was compared based on some socio-demographic factors among the subjects. Subjects residing in urban areas were more prone to malaria compared to those living in rural areas $(X 2=4.957, \mathrm{p}=0.026)$. Figure 3 shows the distribution of Malaria parasitaemia among the subjects based on Residence. Pregnant women with no formal education were more predisposed to malaria infection compared to more educated women with primary, secondary and tertiary education $(X 2=9.040, \mathrm{p}=0.029)$. Figure 4 shows the distribution of Malaria parasitaemia among the subjects based on Educational Status. Pregnant women who did not use insecticide- treated mosquito net was more predisposed to malaria compared to those that do $(X 2=32.105, \mathrm{p}=0.0000)$. Figure 5 shows the distribution of Malaria parasitaemia among the subjects based on Occupational Groups. Pregnant women who are not on prophylactic antimalarial medication were more likely to have malaria compared to those that do $(X 2=18.281, \mathrm{p}=0.0000)$. Figure 6 and Figure 7 shows the distribution of Malaria parasitaemia among the subjects based on use of insecticide treated mosquito nets and use of antimalaria prophylaxis respectively. Figure 8: Blood film from one of the subjects showing several ring forms of P. falciparum. Table 1 shows the Sociodemographic characteristics of subjects.

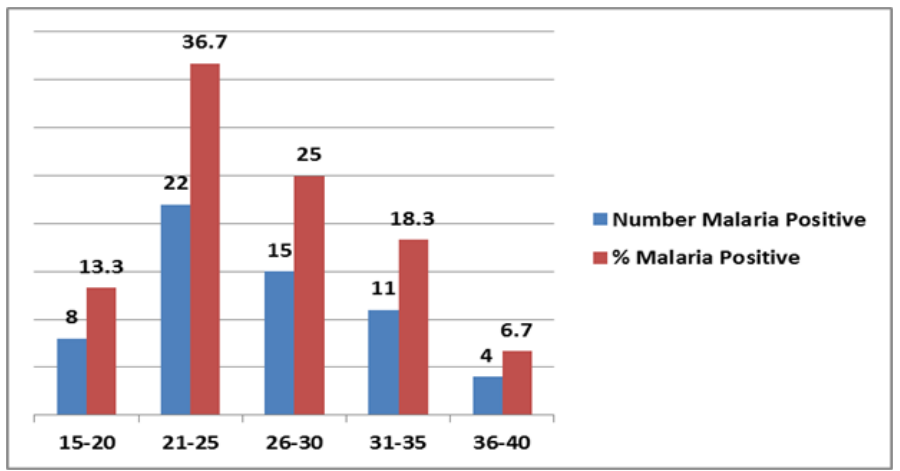

Figure I Distribution of malaria parasitaemia based of age. $X^{2}=2.500, p=0.645$

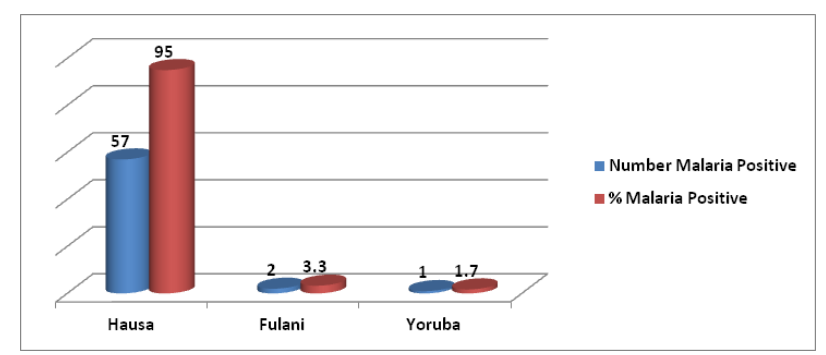

Figure 2 Distribution of malaria parasitaemia among the subjects based on ethnicity.

$X^{2}=5.370, p=0.068$

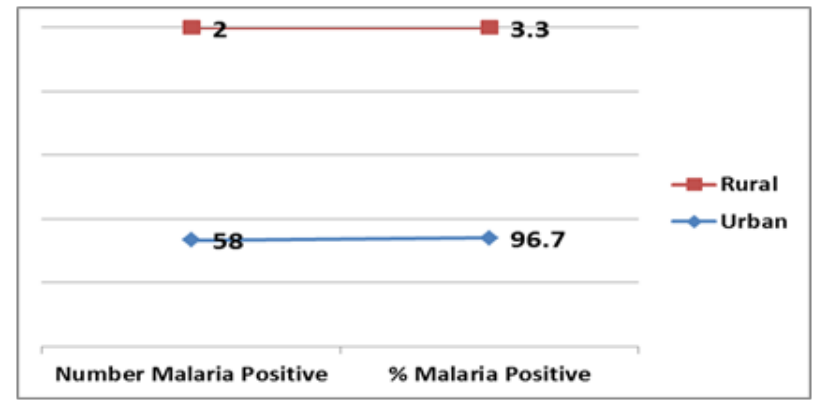

Figure 3 Distribution of malaria parasitaemia among the subjects based on residence.

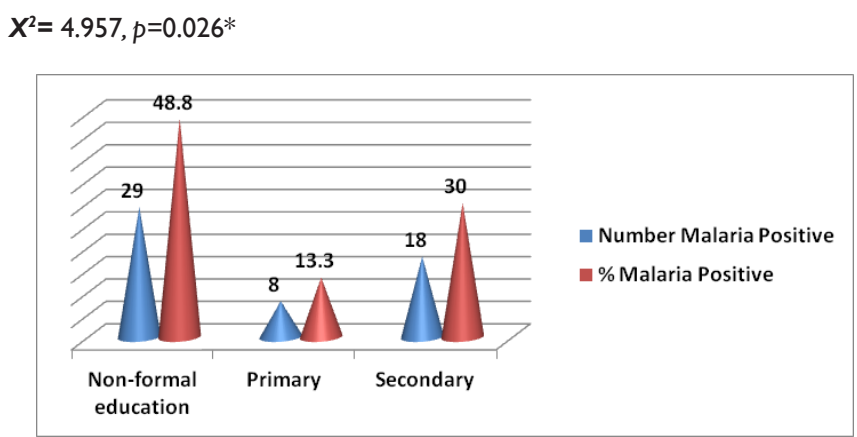

Figure 4 Distribution of malaria parasitaemia among the subjects based on educational status.

$X^{2}=9.040, p=0.029 *$

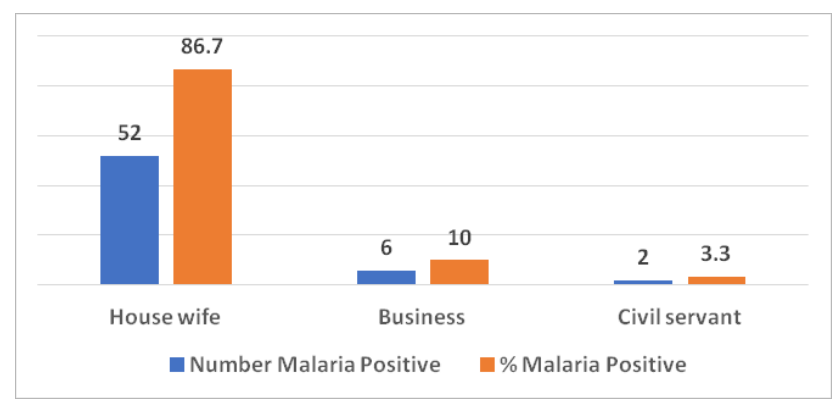

Figure 5 Distribution of malaria parasitaemia among the subjects based on occupational groups.

$X^{2}=2.432, p=0.296$ 


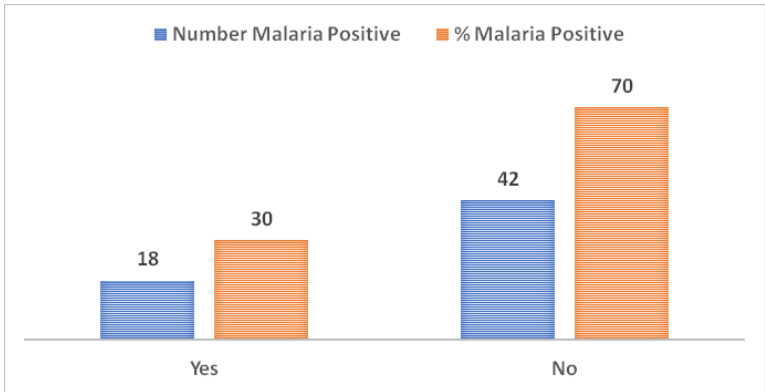

Figure 6 Distribution of malaria parasitaemia among the subjects based on use of insecticide treated mosquito nets.

$X^{2}=32.105, p=0.000^{*}$

Table I The socio-demographic characteristics of subjects

\begin{tabular}{|c|c|c|c|}
\hline Variables & Test $(n=60)$ & $\mathbf{X}^{2}$ & p-value \\
\hline \multicolumn{4}{|l|}{ Age group } \\
\hline $15-20$ & $8(13.3 \%)$ & 2.5 & 0.645 \\
\hline $21-25$ & $22(36.7 \%)$ & & \\
\hline $26-30$ & I5 (25\%) & & \\
\hline $31-35$ & II (I8.3\%) & & \\
\hline $36-40$ & $4(6.7 \%)$ & & \\
\hline \multicolumn{4}{|l|}{ Tribe } \\
\hline Hausa & 57 (95\%) & 5.37 & 0.068 \\
\hline Fulani & $2(3.3 \%)$ & & \\
\hline Yoruba & I (I.7\%) & & \\
\hline \multicolumn{4}{|l|}{ Place of residence } \\
\hline Urban & $58(96.7 \%)$ & 4.957 & $0.026 *$ \\
\hline Rural & $2(3.3 \%)$ & & \\
\hline \multicolumn{4}{|l|}{ Educational status } \\
\hline Non-formal education & $29(48.33 \%)$ & 9.04 & $0.029 *$ \\
\hline Primary & $8(13.33 \%)$ & & \\
\hline Secondary & $18(30 \%)$ & & \\
\hline Tertiary & $5(8.33 \%)$ & & \\
\hline \multicolumn{4}{|l|}{ Occupation } \\
\hline House wife & $52(86.7 \%)$ & 2.432 & 0.296 \\
\hline Business & $6(10 \%)$ & & \\
\hline Civil servant & $2(3.3 \%)$ & & \\
\hline \multicolumn{4}{|c|}{ Use of insecticide treated mosquito net } \\
\hline Yes & $18(30 \%)$ & 32.105 & $0.000 *$ \\
\hline No & $42(70 \%)$ & & \\
\hline \multicolumn{4}{|c|}{ On prophylactic medication } \\
\hline Yes & $26(43.3 \%)$ & $|8.28|$ & $0.000 *$ \\
\hline No & $34(56.7 \%)$ & & \\
\hline
\end{tabular}

Data are presented as mean \pm SEM for age and percentages for others.

Key: t, t-test; $\mathrm{X}^{2}$, chi-square; SEM, standard error of mean; *, statistically significant

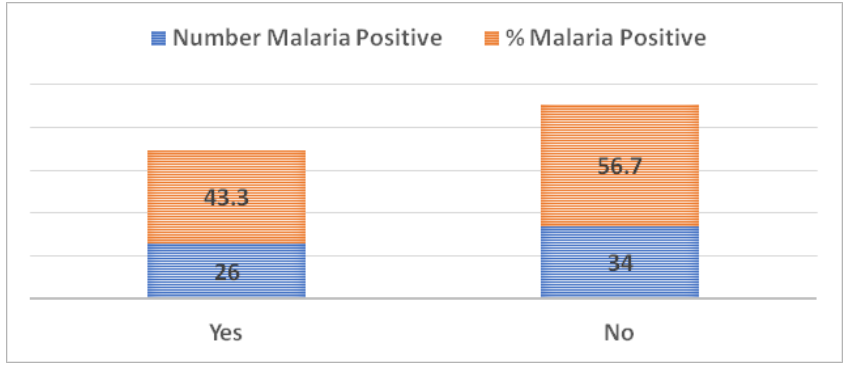

Figure 7 Distribution of malaria parasitaemia among the subjects based on use of antimalarial prophylaxis.

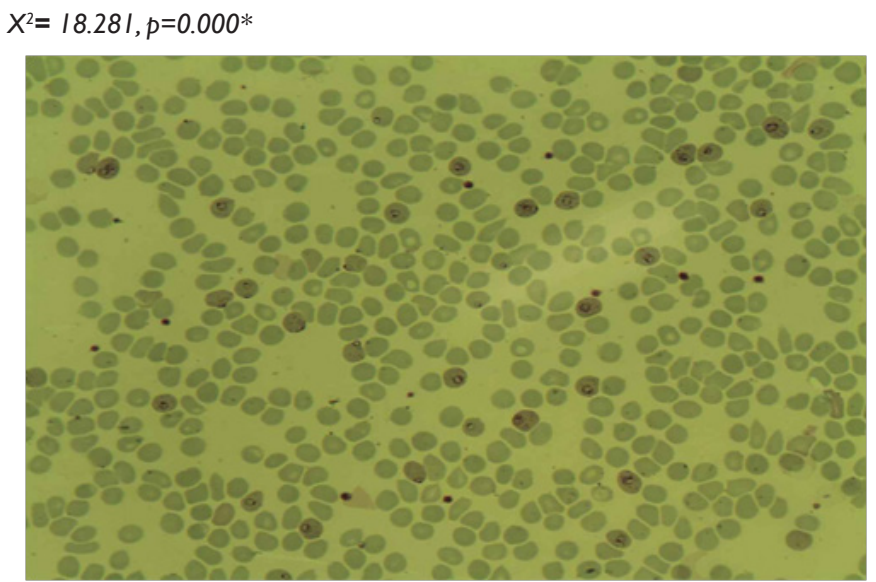

Figure 8 Blood film from one of the subjects showing several ring forms of P. falciparum.

\section{Discussion}

Malaria is a major public health problem in sub-Sahara Africa including Nigeria, where it accounts for more cases of infection and death than other countries in the world and the extent of utilization of malaria preventive measures may impact on the burden of malaria in pregnancy. ${ }^{12}$ This study investigated the socio-demographic risk factors risk factors associated with malaria infection among Pregnant Women of African Descent in Specialist Hospital Sokoto, Nigeria.

We observed that younger women in the age group 21-25 years constituted a significant number of the subjects (36.7\%) compared to older age group 36-40 (6.7\%). This finding is consistent with a previous report by Uneke and Colleagues ${ }^{13}$ which indicated that individuals of age group 20-24 years had the highest prevalence of maternal malaria (52\%) while the least was recorded among those $>40$ years. Our finding is also in agreement with previous report which indicated that pregnant woman of younger maternal age is at a greatest risk of malaria infection as well as having higher parasite densities. ${ }^{14-17}$ Several factors may be responsible for this younger maternal age-related predisposition to malaria; general immune suppression and loss of acquired immunity to malaria. ${ }^{18,19}$

This study has also found that use of insecticide- treated mosquito net has great influence in preventing malaria. Malaria prevalence was significantly higher among women who do not use insecticide treated mosquito nets $(p<0.05)$. This confirms the report of World Health Organization $^{20}$ on the use of insecticide treated mosquito nets as a means to reduce the lethal impact of malaria. Our finding is consistent 
with previous reports in $\mathrm{Kebbi}^{14}$ and Calabar ${ }^{21}$ which indicated the important role that long-lasting insecticide treated nets can play in the prevention of malaria. Our finding collaborates with report by the WHO that the use of ITNs decreases both the number of malaria cases and malaria deaths among pregnant women. ${ }^{22}$

In this study, the level of education was found to have influence on prevention of malaria in pregnancy. Majority of the malaria parasitized subjects had no formal education $(48.3 \%)$, this is followed by those who attained secondary level education (30\%) then followed by women educated to primary education (13\%) and tertiary level education $(8.3 \%)$ of the subjects. This finding is consistent with previous reports in Karachi, India, Maiduguri, Kebbi Sate and Ondo State Nigeria respectively. ${ }^{14,23-25}$ This is suggestive that the level of education can play a role in preventing malaria infection. ${ }^{26,27}$ Our finding is however at variance with a previous report in Lagos which indicated that education was not significantly associated with malaria infection among pregnant women. ${ }^{28}$

The predominant plasmodium specie responsible for all cases of malaria among the subjects was Plasmodium falciparum. This finding is consistent with previous reports ${ }^{29-34}$ in Nigeria which found Plasmodium falciparum as the predominant specie responsible for malaria in Nigeria. Mapping the global distribution of malaria motivated by a need to define populations at risk for appropriate resource allocation, and to provide a robust framework for evaluating its global economic impact, has shown that malaria infection particularly caused by Plasmodium falciparum has been geographically restricted and remains entrenched in poor areas of the world particularly in sub Saharan Africa. ${ }^{35}$

Subjects residing in urban areas were more prone to malaria compared to those living in rural areas $(\mathrm{p}=0.026)$. Our finding is consistent with previous reports ${ }^{36,37}$ which indicated a higher malaria infection in urban compared to rural areas. An evolving major public health challenge seems to be the fact that that urbanization is fast transforming malaria from a rural to an urban disease particularly in poor crowded and rapidly developing urban areas in Nigeria. Several factors promote higher malaria prevalence in urban areas in Nigeria; human migration and the importation of malaria into urban settings, poor housing, suboptimal drainage of Anopheles breeding sites, poor access and use of use of long-lasting insecticide-treated nets (LLINs), poor implementation of indoor residual spraying (IRS), suboptimal implementation of intermittent preventive therapy for pregnant women (IPTp), poor access and use of malaria rapid diagnostic tests (RDTs) and inaccessibility to effective treatments using artemisinin-based combination therapy (ACT).$^{38-40}$ Optimal control of urban malaria depends on accurate epidemiologic and entomologic information about transmission. ${ }^{41}$

We observed in this study that pregnant women who did not use insecticide- treated mosquito net was more predisposed to malaria compared to those that do $(\mathrm{p}=0.0000)$. Use of long- lasting insecticide-treated nets (LLITNs) is one of the key components of malaria prevention and control as recommended by the World Health Organization (WHO) ${ }^{42}$ The nets reduce human contact with mosquitoes, thus leading to a significant reduction in the incidence of malaria, associated morbidity, and mortality; as well as in the adverse effects during pregnancy in areas of intense malaria transmission. ${ }^{43}$ Our finding is consistent with previous reports which indicates that the use of insecticide-treated nets (ITNs) for malaria vector control is effective in controlling malaria attacks in pregnant women. ${ }^{44-46}$ An estimated 27 million ITNs are required annually to effectively protect these most vulnerable groups from malaria infection in Nigeria. ${ }^{47}$ Unfortunately, few people in high-risk region have access and use ITNs. This is a public health challenge that requires prompt attention. Previous report however indicates that the challenge does not necessarily possess an ITN but low utilization in Nigeria. ${ }^{48}$

In this study, we observed that pregnant women who are not on prophylactic antimalarial medication were more likely to have malaria compared to those that do $(\mathrm{p}=0.0000)$. Our finding is consistent with a previous report ${ }^{49}$ which suggesting the continuation of IPTp to reduce ppregnancy-associated malaria morbidity. An evidenced based way of controlling malaria in pregnancy is the administration of intermittent preventive treatment with sulfadoxine-pyrimethamine during pregnancy (IPTp-SP). WHO recommends IPTp with sulfadoxinepyrimethamine (IPTp-SP) in all areas with moderate to high malaria transmission in Africa. ${ }^{50}$ This consists of a full therapeutic course of antimalarial medicine given to pregnant women at routine prenatal visits, regardless of whether they are infected with malaria or not. Intermittent preventive treatment reduces incidences of maternal malaria episodes, maternal and foetal anaemia, placental parasitaemia, low birth weight and neonatal mortality. Evidenced based best practice in Mali of Intermittent preventive treatment in pregnancy (IPTp) with 3 or more doses of sulfadoxine-pyrimethamine (SP) has been shown to prevent malaria in pregnant women living in highrisk areas. ${ }^{51}$ Despite the fact that intermittent preventive therapy in pregnancy (IPTp) with sulfadoxine-pyrimethamine (IPTp-SP) is a clinically-proven method to prevent the adverse outcomes of malaria in pregnancy for the mother, her foetus, and the neonates, progress towards implementation of universal coverage remains suboptimal with widespread regional and socioeconomic disparities in the utilization of this highly cost-effective intervention. ${ }^{52}$

\section{Conclusion and recommendations}

\section{Conclusion}

This study has shown that Plasmodium falciparum is the predominant malaria specie among plasmodium parasitized women in Sokoto, North Western Nigeria. Socio-demographic factors (residence, illiteracy, non-availability and non-compliance to use of long-lasting insecticide-treated mosquito nets and lack of access to antimalaria prophylaxis during pregnancy) are factors contributing to prevalence of malaria among pregnant African women. There is need to routinely test pregnant women presenting to antenatal clinic particularly with febrile illness for malaria. There is need for the Nigerian government to invest in girl child education and increased awareness about malaria preventive measures, early and regular antenatal clinic attendance as well as universal provision of long insecticide treated mosquito nets and prophylaxis for all pregnant women to reduce the incidence of malaria and its associated morbidities and mortalities.

\section{Acknowledgments}

None.

\section{Conflicts of interest}

The authors declare there is no conflict of interests. 


\section{References}

1. Dellicour S, Tatem AJ, Guerra CA, et al. Quantifying the number of pregnancies at risk of malaria in 2007: a demographic study. PLoS Med. 2010;7(1):e1000221.

2. WHO. Guidelines for the treatment of malaria. Geneva: World Health Organization; 2006.

3. Mockenhaupt FP, Ulmen U, von Gaertner C, et al. Diagnosis of placental malaria. J Clin Microbiol. 2002;40(1):306-308.

4. Desai M, ter Kuile FO, Nosten F, et al. Epidemiology and burden of malaria in pregnancy. Lancet Infect Dis. 2007;7(2):93-104.

5. Agomo CO, Oyibo WA, Anorlu RI, et al. Prevalence of malaria in pregnant women in Lagos, South-West Nigeria. Korean J Parasitol. 2009;47(2):179-183

6. Tillotson GS. Foreword: 10-year special edition. J Expert review of antiinfective therapy. 2012;10(11):1219-1220.

7. Amuta E, Houmsou R, Wama E, et al. Malarial Infection among antenatal and maternity clinics attendees at the federal medical centre, Makurdi, Benue State, Nigeria. Infect Dis Rep. 2014;6(1):5050.

8. Oyefabi A, Sambo M, Sabitu K. Effect of primary health care workers training on the knowledge and utilization of intermittent preventive therapy for malaria in pregnancy in Zaria, Nigeria. Journal of Medicine in the Tropics. 2015;17(1):4-11.

9. Umaru ML, Uyaiabasi GN. Prevalence of malaria in patients attending the general hospital Makarfi, Makarfi Kaduna-State, North-Western Nigeria. American Journal of Infectious Diseases and Microbiology. 2015;3(1):1-5.

10. NPC/FGN. Nigerian population commission, federal republic of Nigeria. Special FGN Gazette No. 23 on the 2006 Population Census; 2006.

11. Udomah FP, Isaac IZ, Lukman I, et al. Plasmodium parasitemia among pregnant women attending antenatal clinic in Sokoto, North western Nigeria. Journal of Nursing Sciences. 2015;1(1):9-14.

12. Saidu AY, Sadiya H, Dikwa MA, et al. Detection of plasmodium species among pregnant women attending antenatal care. IOSR Journal of Dental and Medical Science. 2015;14(11):61-66.

13. Uneke CJ, Sunday-Adeoyeb I, Iyarec FE, et al. Impact of maternal Plasmodium falciparum malaria and haematological parameters on pregnancy and its outcome in southern Nigeria. Journal of Vector Borne Diseases. 2007;44(4):285-290.

14. Fana SA, Bunza MD, Anka SA, et al. Prevalence and risk factors associated with malaria infection among pregnant women in a semi-urban community of north-western Nigeria. Infect Dis Poverty. 2015;4:24.

15. Agomo CO, Oyibo WA. Factors associated with risk of malaria infection among pregnant women in Lagos Nigeria. Infect Dis Poverty. 2013;2:19.

16. Marielle KBA, Denisa EIC, Modeste MM, et al. Prevalence of Plasmodium falciparum infection in pregnant women in Gabon. Malar J. 2003;2:1-17.

17. Adam I, Khamis AH, Elbashir MI. Prevalence and risk factors for Plasmodium falciparum malaria in pregnant women of eastern Sudan. Malar J. 2005;4:18.

18. Beeson JG, Duffy PE. The immunology and pathogenesis of malaria during pregnancy. Curr Top Microbiol Immunol. 2005;297:187-227.

19. Staalsoe T, Shulman CE, Buhner JN, et al. Variant surface antigenspecific IgG and protection against clinical consequences of pregnancyassociated Plasmodium falciparum malaria. Lancet. 2004;363:283-289.

20. World Health Organization. World malaria report fact sheet. 2011.
21. Iwuafor AA, Egwuatu CC, Nnachi AU, et al. Malaria Parasitaemia and the use of insecticide-treated nets (INTs) for malaria control amongst under-5 year old children in Calabar, Nigeria. BMC Infect Dis. 2016;16:151.

22. Jombo GTA, Mbaawuaga EM, Ayegba AS, et al. How far have we rolled back malaria on the African continent nine years down? The burden of malaria among pregnant women in a semi-urban community of northern Nigeria. J Med Med Sci. 2010;1(6):235-241.

23. Ali SS, Karim N, Billoo AG, et al. Association of literate mothers with malnutrition under three years of age in rural areas of district Malir, Karachi. J Pak Med Assoc. 2005;55(12):550-553.

24. Hamidu JL, Salami HA, Ekanem AU, et al. Prevalence of protein energy malnutrition in Maiduguri, Nigeria. African Journal of Biomedical Research. 2003;6(3):123-125.

25. Akanbi FR. Socio-economic and demographic impact on malaria prevalence in Akoko South-west of Ondo state, Nigeria. International Journal of Infectious Diseases. 2016;45:214.

26. WHO. Implementation of the Global malaria control strategy. Report of WHO study Group Geneva: World Health Organization; 1993.

27. Legesse Y, Tegegn A, Belachew $T$, et al. Knowledge, attitude and practice about malaria transmission and its preventive measures among households in urban areas of Assosa zone, Western Ethiopia. Ethiop $J$ Health Dev. 2007;21(2):2.

28. Agomo CO, Oyibo WA. Factors associated with risk of malaria infection among pregnant women in Lagos Nigeria. Infect Dis Poverty. 2013;2:19.

29. Jeremiah ZA, Jeremiah TA, Emelike FO. Frequencies of some human genetic markers and their association with Plasmodium falciparum malaria in the Niger Delta, Nigeria. J Vector Borne Dis. 2010;47(1):1116.

30. Erhabor O, Adias TC, Hart ML. Effects of falciparum malaria on the indices of anaemia among pregnant women in the Niger Delta of Nigeria. Journal of Clinical Medicine and Research. 2010;2(3):035-041.

31. Erhabor O, Jeremiah ZA, Adias TC, et al. Thrombocytopenia in plasmodium parasitized pregnant women in the Niger Delta of Nigeria. Pathology and Laboratory Medicine International. 2010:21-25.

32. Adigun $\mathrm{AB}$, Gajere $\mathrm{EN}$, Oresanya $\mathrm{O}$, et al. Malaria risk in Nigeria: Bayesian geostatistical modelling of 2010 malaria indicator survey data. Malar J. 2015;14:156.

33. Omolade Olayinka Okwa. Nipping the malaria vectors in the bud: focus on Nigeria. Intech Open. 2019.

34. Erhabor O, Azuonwu O, Frank-Peterside N. Malaria parasitaemia among long distance truck drivers in the Niger Delta of Nigeria. Afr Health Sci. 2012;12(2):98-103.

35. Robert W Snow, Carlos A Guerra, Abdisalan M Noor, et al. The global distribution of clinical episodes of Plasmodium falciparum malaria. Nature. 2005;434(7030):214-217.

36. Wilson ML, Krogstad DJ, Arinaitwe E, et al. Urban malaria: understanding its epidemiology, ecology, and transmission across Seven diverse ICEMR network sites. Am J Trop Med Hyg. 2015;93(3):110-123.

37. Cator LJ, Thomas S, Paaijmans KP, et al. Characterizing microclimate in urban malaria transmission settings: a case study from Chennai, India. Malar J. 2013;12:84.

38. Morakinyo OM, Balogun FM, Fagbamigbe AF. Housing type and risk of malaria among under-five children in Nigeria: evidence from the malaria indicator survey. Malar J. 2018;17(1):311.

39. Nkumama IN, O'Meara WP, Osier FHA. Changes in malaria epidemiology in Africa and new challenges for elimination. Trends Parasitol. 2017;33(2):128-140. 
40. Pinder M, Conteh L, Jeffries D, et al. The RooPfs study to assess whether improved housing provides additional protection against clinical malaria over current best practice in The Gambia: study protocol for a randomized controlled study and ancillary studies. Trials. 2016;17(1):275.

41. Wilson ML, Krogstad DJ, Arinaitwe E, et al. Urban malaria: understanding its epidemiology, ecology, and transmission across Seven diverse ICEMR network sites. Am J Trop Med Hyg. 2015;93(3):110-123.

42. WHO. World malaria report. Geneva: World Health Organization; 2008:1-183

43. TerKuile FO, Terlouw DJ, Philips-Howard PA, et al. Reduction of malaria during pregnancy by permethrin-treated bed nets in an area of intense perennial malaria transmission in western Kenya. Am J Trop Med Hyg. 2003;64(2):50-60.

44. Nwagha TU, Nwagha UI, Dim CC, et al. Benefit incidence analysis of free insecticide treated nets distribution in urban and rural communities of Enugu state, South East Nigeria. Niger J Clin Pract. 2014;17(2):168173.

45. Rashed S, Johnson H, Dogier P, et al. Economic impact of febrile morbidity and use of permethrin-Impregnated bed nets in a malarious Area Study of demographics, morbidity and household expenditure associated with febrile morbidity in the Republic of Benin. Am J Trop Med Hyg. 2000;62(2):173-180.

46. Macintyre K, Keating J, Okbaldt YB, et al. Rolling out insecticide treated nets in eritrea: examining the determinants of possession and use in malarious zones during the rainy season. Trop Med Int Health 2006;11(6):824-833
47. Onwujekwe O, Hanson K, Fox-Rushby J. Inequalities in purchase of mosquito nets and willingness to pay for insecticide treated nets in Nigeria: Challenges for malaria control interventions. Malar J. 2004;3:6

48. Afolabi BM, Sofola OT, Fatunmbi BS, et al. Household possession, use and non-use of treated or untreated mosquito nets in two ecologically diverse regions of Nigeria--Niger Delta and Sahel Savannah. Malar J. 2009;8:30.

49. Cisse M, Awandare GA, Soulama A, et al. Recent uptake of intermittent preventive treatment during pregnancy with sulfadoxine-pyrimethamine is associated with increased prevalence of Pfdhfr mutations in BoboDioulasso, Burkina Faso. Malar J. 2017;16(1):38.

50. WHO. A strategic framework for malaria prevention and control during pregnancy in the African region. Brazzaville: World Health Organization, Regional Office for Africa; 2004.

51. Diarra S, Konaté D, Diawara SI, et al. Factors Associated with Intermittent Preventive Treatment of Malaria During Pregnancy in Mali. J Parasitol. 2019;105(2):299-302.

52. Yaya S, Uthman OA, Amouzou A, et al. Use of Intermittent Preventive Treatment among Pregnant Women in Sub-Saharan Africa: Evidence from Malaria Indicator Surveys. Trop Med Infect Dis. 2018;3(1). 